\title{
Research on the Application of Artificial Intelligence in Human Resource Management
}

\author{
Fengxiang Jiang, Changjun Du, Tongtong Fu, Runting Xiao \\ Xi'an Peihua University, Xi’an Shaanxi, 710125
}

Keywords: artificial intelligence; human resources management

\begin{abstract}
The emergence of artificial intelligence provides unlimited possibilities for the improvement of efficiency and quality of human resource management in various industries, and new changes will take place in the field of human resources management. This paper first describes the connotation and characteristics of artificial intelligence, then analyzes the influence of artificial intelligence on the efficiency of human resources management, and then analyzes the bottleneck problem in the application of artificial intelligence in human resources management. Finally, it tries to explore the specific ideas of artificial intelligence in the practice of human resources management.
\end{abstract}

\section{Introduction}

Premier Li Keqiang put forward artificial intelligence in his government work report at the fifth session of the 12th National people's Congress of the People's Republic of China on March 5, 2017. Artificial intelligence will be used in a large number of industries, to promote the transformation and reform of various industries. Will human resources be replaced by artificial intelligence? Can human resource management make full use of artificial intelligence to improve work efficiency and quality? This paper will study the influence and bottleneck of artificial intelligence on human resource management in order to explore the application path of artificial intelligence in human resource management.

\section{The connotation and characteristics of artificial intelligence}

Definition of Artificial intelligence is a widely accepted and popular in this paper, artificial intelligence is to make the behavior of machines looks like the intelligent behavior of human beings, reflecting a certain degree of intelligence. Most of the definitions of artificial intelligence can be divided into four categories: machines think like man, act like man, think rationally and act rationally.

\section{The influence of artificial Intelligence on the efficiency of Human Resource Management}

\subsection{The influence of artificial Intelligence on the efficiency of Human Resource Planning}

The HR mastering the data varies from person to person, and because of the complexity and difficulty of the work nature and content, it has a lot of influence on the proficiency of HR in the traditional human resource planning. Human resource planning by HR often leads to the deviation of the precision of planning and the forecast of personnel demand due to the insufficient degree of data information and mastery. HR needs huge cost of manpower, time and so on in the process of human resource planning. In particular, the internal and external data collection and personnel communication are hindered and the task is very difficult. Through AI's extensive algorithms and machine learning tools, based on the big data analysis, we can quickly find and match the information that conforms to the characteristics of the enterprise and the development trend of the industry, optimize the data and forecast the future demand of the enterprise. An accurate analysis of the existing organization's human resources can be obtained, and HR can make a quick judgment based on this data. Artificial intelligence takes the place of HR to make the basic collection and 
collation work, and gives appropriate suggestions and data analysis.

\subsection{The effect of artificial Intelligence on recruitment efficiency}

HR candidates not only look on their professional qualities, but most of them are affected by the candidates' appearance, words and deeds, clothing and so on In traditional recruitment, which inevitably affect the accuracy and objectivity of the interview. Although some enterprises use the most professional and authoritative quality evaluation model, they cannot avoid the selection of people who are not suitable for the enterprise, and there will be cases of recruitment failure. In the future, artificial intelligence will not only be applied in the field of human resources, but will have its unique side to human behavior, psychology and so on. A wide range of algorithms and intelligent learning tools based on artificial intelligence can be used to judge candidates more directly and objectively, to find the best people suitable for the enterprise, and to analyze the candidates in the process of quality evaluation. The evaluation model is more accurate based on big data and AI analysis. AI can not only eliminate discrimination, eliminate prejudice, eliminate emotional factors, but also quickly screen resumes and match personnel.

\subsection{The effect of artificial Intelligence on training efficiency}

The enterprise personnel training often costs high output and investment, often does not get the very good return, and HR in the training process will make the trained personnel produce a lot of judgment and psychological defense because of the training speaker's behavior, the appearance manner, Professional knowledge. On the contrary, an intelligent machine has good stability to the psychology of ordinary people, and it is not easy to produce hostile influence. Artificial intelligence can be through training, as the keynote speaker of enterprise training. Not only can we find a variety of models suitable for enterprise training through a large amount of information data, but also can track and adjust the training situation of personnel at any time in the course of training, and feedback effect, so as to maximize the input and output of enterprise training. The intervention of artificial intelligence can not only replace the trainer as the keynote speaker and improve the training effect, but also can help HR to train the enterprise staff and be the assistant of the trainer. On the other hand, artificial intelligence can also play a convenient, ready, and "housekeeper" role, so that each employee has an artificial intelligence trainer, more with a combination of private customized trainers. It not only saves the cost greatly, but also improves the effect of training.

\subsection{The effects of artificial intelligence on performance and payment efficiency}

Traditional performance management has the influence of attitude and the existence of informal organizations among members because of the subjective emotional exchange between people, so that the performance appraisal work cannot be completely objective. Artificial intelligence can make the record data more accurate, more objective, to reduce the daily performance appraisal error caused by the psychological loss of employees. Moreover, the analysis of enterprise development status data, industry performance data, urban development status and other data can make artificial intelligence can formulate an objective and fair performance appraisal system in the process of formulating performance standards. And it can be adjusted in real time through data analysis, so that the performance of the whole enterprise is no longer solidified, more suitable for employees, more incentive, improve the enthusiasm of employees, reduce the unnecessary cost of enterprise waste.

\subsection{The effects of artificial Intelligence on the efficiency of Labor Relations Management}

The traditional employee relationship is more engaged in repetitive work, such as: social security payment, process processing, leave procedures, employee contract disputes and so on. Artificial intelligence not only simplifies and facilitates the work, through machine learning tools and algorithms. It helps to choose the best scheme directly, provide the enterprise and employee, and make both sides establish reasonable labor relations under the objective and fair conditions. In the process of continuously updating the data, artificial intelligence has made more good suggestions and integration for the development of enterprises and the career planning of employees. The 
healthy development of employee labor relations can not only enable enterprises to reduce the costs borne by labor disputes. It can also establish an integrated corporate culture of employees, so that with the help of artificial intelligence, the corporate culture can play a role of culture, and the organizational and personal goals of the enterprise can be integrated, so that the enterprise has a more sense of social responsibility. Employees are more cohesive and happy, improve motivation and work efficiency.

\section{The bottleneck of artificial intelligence application in human resource management}

\subsection{The bottlenecks of artificial intelligence in human resource planning}

Strategic planning is needed in human resource management. Human resource manager will analyze and summarize the past work, and have certain foresight to the future development trend of enterprise when making strategic plan. The premise of artificial intelligence work is that HR is required to do a good job of judgment, the specific work of sensibility and innovation or the need for managers to complete.

\subsection{The bottleneck of artificial Intelligence in Human Resource recruitment}

When enterprises recruit new employees, artificial intelligence can select résumés scientifically, quickly and accurately. But in the interview process, HR recruiters will evaluate the candidates with their own work experience, and test their ability to react and adapt to the series of problems that may arise in their work.

\subsection{The bottlenecks of artificial intelligence in human resources training}

The cost of artificial intelligence may not be high, but the training of artificial intelligence is limited to some theoretical knowledge and workflow in the training of new employees, unable to be a trainee's substitute, rich in content, employees will only find it boring. And the promotion and promotion of corporate culture in the training must be based on the conviction and advocacy of the enterprise personnel. When the new employees are nurtured by the corporate culture, artificial intelligence cannot describe and interpret the enterprise culture in a vivid way. There is no experience of personal experience. Employees cannot open their hearts to listen and think when faced with a machine, so the communication between people and machines is far more effective than the communication between people and machines.

\subsection{The bottleneck of artificial Intelligence in compensation performance Management}

Performance interview is the work of deep communication with employees in the process of performance management. Artificial intelligence cannot achieve emotional communication and emotional communication, cannot stand on the perspective of staff and leadership two-way communication. Artificial intelligence is limited to systematic accurate judgment in basic transactional work. once there are a series of problems in the implementation of artificial intelligence, the enterprise cannot attribute the responsibility to a computer, and the same person will also have the idea of evading responsibility and pass the responsibility he should take on to the machine in terms of responsibility and authority.

\subsection{The artificial Intelligence in dealing with bottlenecks in employee relations}

Dealing with employee relations requires us HR managers to go deep into the staff's work and understand their ideas in human resources management. Modern human resource management pays more attention to humanized management. Through the deep communication and infection of human resource managers, employees can obtain satisfaction and existence in psychology, and thus have a strong dependence on the company. However, if artificial intelligence is used in communication, it will make employees feel less valued, and will not get the affinity of communication between people, thus they will not get the real ideas of employees. Nor can the person's emotion be digitized and programmed.

In short, artificial intelligence can help HR do some basic work, improve their work efficiency 
and work scientifically and accurately. But when the concrete human resource management is implemented, artificial intelligence cannot be as flexible as human management, it can only assist the work of HR.

\section{The concrete thinking of artificial intelligence in the practice of human resource management}

\subsection{To digitize human resources management}

It is necessary to establish the enterprise artificial intelligence independent learning knowledge base and the human resource management intelligent MIS system, the reserve core information data, the analysis enterprise strategy, becomes the partner of enterprise, lets the human resources management pattern digitize and digitize.

\subsection{To automate and process simple and repetitive work}

According to the current situation of the enterprise and the enterprise industry data, the artificial intelligence can analyze the personnel recruited by the enterprise, the performance appraisal standard and so on, and directly predict how and from where the enterprise needs to recruit people. Taking recruitment as an example, through the information data analysis of artificial intelligence, in the shortest time to screen a number of complex resumes, and on this basis can also be more in-depth analysis of the candidate's various data, more accurate judge whether the post is a match, whether belongs to the enterprise suitable person. Artificial intelligence instead of junior interviewers, the most accurate recruitment work, is to give enterprises more advice. Machine vision, fingerprint recognition, faces recognition and performance management is applied in human resource management.

\subsection{To optimize the organizational structure of an enterprise}

AI system continuously optimizes its internal and organizational structure to form self-organization through data accumulation and algorithm analysis. Modern artificial intelligence systems have been able to identify human emotions in a variety of ways, and after constantly upgrading data; they can quickly identify employee pressure and organizational synergy. And if the combination efficiency of the enterprise group and the staff is low, the new group can be reorganized and assigned to the personnel, so there will be no boundaries between the departments, the group and the department of the enterprise will be more efficient, and the organizational structure will change significantly.

\section{Conclusion}

In short, in the era of artificial intelligence, human resource management needs to speed up the digital transformation, pay attention to the use of data, until the comprehensive upgrade analysis, the whole process is not overnight, but a continuous iterative, evolving process. The application of artificial intelligence will greatly reduce the cost of the enterprise, reduce the substitutable basic work of HR, help HR enhance the analysis data, become the strategic partner of the enterprise, and the work and mode of human resource management will be changed completely.

\section{References}

[1] Yinlong Li, Xiaohong Wu. Analysis on the Influence of Artificial Intelligence on Human Resource Management [J]. Human Resources Management, 2018 (3): 400-401.

[2] Yao Shi. Study on the Value Adjustment of Human Resources Management in Public Sector in the Age of artificial Intelligence [J]. Journal of Nantong Institute of Shipping Technology, 2018 (2): 20-23

[3] Liang Wang. Using Artificial Intelligence to improve the Level of Human Resources 
Management [J]. Sinopec in China, 2017 (7): 53-54.

[4] Xiangyu Qi. Artificial Intelligence HR, is taking place in the Future-Dialogue Yi Qifei (Beijing) Human Resources Technology Co., Ltd Chairman Zhang Zhaolei [J]. Human Resources, 2017 (12): $12-18$

[5] Mei Lin, Xiuhua Ling, Chen Chao. Will artificial intelligence take HR's Job? [J]. Modern Commerce, 2018 (8): 61-63 